\title{
An Innovative Face Emotion Recognition-based Platform by using a Mobile Device as a Virtual Tour
}

\author{
Luigi Patrono, Member, IEEE, Luca Podo, and Piercosimo Rametta
}

\begin{abstract}
Emotions are the base of human evolution. They help us to survive and to face up all problems of our life. Without emotions human evolution was not possible and we would be in caves. Nowadays, emotions are a very important aspect in different field not only in psychology. They are very important to understand human mind and decision-making process. Emotional tourism is an example of a new way to use emotions analysis to predict how traveler could enjoy destinations, leisure and tourism services. It provides support for the suitability of emotions as a segmentation variable. In this field emotions are used to create a most deep experience from the begin of a travel to each steps of the journey. They help tourism to make traveler the protagonist of his travel and not just a spectator. In this paper, we are going to show an app which predicts a travel destination based on user's mood and facial expressions to specifics visual and auditory trigger to encounter his reactions. This app uses different technology linked together to make this solution versatile and dynamic. It implements different technology modules to perform facial and mood analysis, capturing the image, store image and show all trigger to the user. By adopting this solution is possible to easily upgrade the app and each module can be changed with no large problem adapting it to the current version of the app.
\end{abstract}

Index Terms - Emotion Analysis, Machine Learning, Cloud, Image recognition, App, Emotional Tourism.

\section{INTRODUCTION}

$\mathrm{E}$ MOTIONS are at the base of the development of human beings, in terms of progress and evolution. They are a vital instrument of the evolution, since they do not depend on cultural aspects and they are not a voluntary expression. Indeed, emotions are spontaneous manifestations of involuntary expressions to internal or external stimulations. Emotions are a psychophysics response to internal or external changes, that can be perceived as positive or negative.

Many academic research activities are focused on the study

Manuscript received December 19, 2018; revised March 5, 2019. Date of publication April 24, 2019. Date of current version June 3, 2019.

Authors are with the Innovation Engineering Department, University of Salento, Via per Monteroni, 73100, Lecce, Italy.

Luigi Patrono is the corresponding author (e-mail: luigi.patrono@unisalento.it).

Digital Object Identifier (DOI): 10.24138/jcomss.v15i2.675 of emotions, because they allow to analyse and study how a human being will react to a specific stimulation.

In academic literature there are two main groups of emotions:

- Basic emotions [1] developed since childhood and related to survivability, to establish relations and to carry out basic activities. More precisely they are: joy, anger, contempt, fear, sadness, disgust and surprise.

- Complex emotions related to the development of knowledge and to the communication within the social environment.

The main purposes of emotions are:

- To react and to get ready to emergency: they allow us to understand dangerous situations and to get ready to action.

- To communicate with others: they allow us to improve and strengthen emotional connection with other people.

- To communicate with yourself: they allow us to overcome mental and environmental obstacles and to get motivated.

Emotional stimulations are generated from different sources, mainly related to the human five senses. For example, what we eat, touch, listen to or look at can stir up our emotions. Images play an important role about this topic, because they can be used to convey mood-related information to others.

Nowadays, emotions related to images are a very important trend topic in fields like tourism and marketing. Several studies showed how an emotional campaign is more effective than a normal campaign, to transmit a message about a brand or a product [2]. Emotions related to images are a very important aspect for a lot of major companies to improve human-computer interaction and to predict and guide the people's decision-making process. The importance of emotional campaigns, using motivational text or evocative images, relies on the fact that a consumer decides whether to buy a product or not on the base of its first emotional impact, and then the rational part of the brain comes into play. This rational part of the brain validates the decision that has being 
taken. Therefore, all these analyses of our emotions to certain visual stimuli are very important for the decision-making and the prediction of future behaviour of groups of individuals towards a new product. Examples of such approach are two famous emotional campaigns like "Just do It" carried out by Nike [3], and "Real Beauty" carried out by Dove [4].

Human emotional response to an image is a multidimensional variable. Each response depends on different causes, such as colours, brightness, movement and human presence. Recently Facebook, one of the most popular social networks, introduced the functionality to share users' emotions about all kind of images, videos or posts on their platform. By using this easy way to collect users' emotions, they can conduct an emotional analysis of their users.

Images create a spontaneous response, because human being links what he/she is seeing to his/her previous experiences. This ancestral emotional mechanism can be exploited during the process of arranging a touristic travel or holiday, since the moment the user is defining the target destination. A trip is not a simple travel, but it is a new kind of experience, in which travelers will meet new people and will discover new cultures. In recent years, the so-called "emotional tourism" approach is emerging. It refers to the new trend of using emotions analysis to predict how traveler could enjoy destinations, leisure and tourism services. It provides support for the suitability of emotions as a segmentation variable [5] in decision making processes. In this scenario, emotions are very important from the early stages, and thanks to new technologies, such as facial expression recognition, it will be possible to find the best destination based on mood, nature and wanderlust of traveler. According to a study carried out by Ohio State University and published on Proceedings of National Sciences [6], through facial expression recognition is possible to find which emotion is feeling the user, distinguishing among 21 different emotions. Emotions will drive the user during the travel planning, suggesting and influencing what to do. The use of such technologies in tourism is an important tool, since it provides to tourists and to tourism operators a lot of data about decision making, greater mobility and finest destinations [7].

The main advantages of using these technologies in tourism are:

- Support to decision making process

- Development of new services and products

- Improve relations with tourists

- Cheaper and faster data-processing

- Niche recommender system

Soon, these technologies will be able to read real-time and reliable data about user mood, allowing a better humanmachine interaction and highly customized and effective travel solutions.

In this context, this paper deals with the design and implementation of a novel software architecture for the provision of highly customized emotional tourism solutions, by exploiting the user mood during the travel definition phase. The system is mainly based on a smartphone application, called "ETravel", which collects data about user facial expressions while he/she is subjected to visual stimuli related to the journey he/she is preparing. The system will analyse and extract emotions from each photo taken for all trigger images. If the mood is positive, the system will generate a label characterizing the image. Each label is used to find all kind of travel destinations that should be very close to user's preferences. By performing an alfa test on $\mathrm{CK}+$ database, the app was able to return a correct destination based on the kind of expression we passed to the camera. Also, the test with subject returns us some positive results, but we got some problems to identify the micro-expressions. This result is based on the first version of the app, and in future with a better FER module the app could return better results.

\section{StATE OF THE ART}

By using emotion recognition through face images, it is possible to develop some innovative solutions in several application scenarios, as an example, in the field of medicine, tourism, marketing or consumer apps. In each field there are one or more solutions that use emotion recognition to create a better human machine interaction.

In the medicine field, the "CaptureMyEmotion" mobile app [8] has been developed to help autistic children. This app enables children to interact with a mobile phone to take photos, videos or sounds. It uses a Sympathetic Nervous System (SNS) to measure arousal (high or low level of emotion) and facial analysis for valence (positive or negative emotion). This app is used to assign emotions to pictures or a sound taken by children. This can help autistic children to improve their emotions learning. This solution is based on Affectiva [9] technology. Affectiva Emotion Artificial Intelligence (AI) measures unfiltered and unbiased facial expressions of emotion, using any optical sensor or just a standard webcam. It can identify human face in real time or using an image or a video. It provides a solution to detect 7 emotions, 20 expressions and 13 emojis and includes classifiers for age, gender and ethnicity. It uses an emotions database with more than 6.5 million faces from 87 countries analysed.

Another application context of emotion recognition is related to the monitoring of patients with mood disorders. In [10], authors deal with a desktop and mobile app which analyses in real time user's mood and predicts a song to play. This could provide a song for mood enhancement. In this work, the app takes a photo of the user every $\mathrm{X}$ frame, equals to the frame rate of the camera. It makes a face filtering operation and then it analyses emotions. A music player will fade in or fade out a song based on mood prediction to provide relief. To test and train facial and emotions recognitions authors used FACS [11]. FACS is a set of facial muscle movements that correspond to displayed emotion. It is used to parameterize facial behaviour and motions according to muscle actions. This app has only some limitations when there is some movement and side views in the video. Over 20 degrees of frontal views, the efficacy in recognition drops. They used OpenCV for mobile and desktop app to detect and 
to process face image captured by a camera.

The system described in [12] is an example of developing a solution to predict and to study customer purchasing behaviour. It is about a novel framework to analyse facial expression of a customer in relation to a product. In this case, emotional conditions are always present and influence every stage of decision-making. This app takes a sequence of images from key frames in different periods and classifies user's emotions. From these results, an opinion on the impact is formulated. In order to detect faces and recognize emotions, this solution is based on a classification by SVM. SVM are supervised learning models with associated learning algorithms that analyse data used for classification and regression analysis.

"Empathic Dairy" [13] is another example of app that uses this kind of technology. It is a common personal app diary, which analyses photo and video of the user and it provides an emotional feedback of the session. This app uses "Emotion from Face" module embedded into Java Native Interface (JNI) to perform face emotion analysis. It also uses text emotion detection to provide a better emotion detection of the user.

In [14] authors deal with an innovative solution to measure emotions by customer's perceptions of hotel spaces. They try to stimulate customer's reaction by using real and virtual hotel scenery to detect which one is more effective. To trigger a customer's reaction, they used a video with background music for the virtual tour. A webcam was used to collect customer's photo. To perform a real tour, they used presidential rooms and all participants wore an element with a GoPro to detects facial expressions. Finally, all data collected have been analysed by FaceReader software, a facial expression recognition system that identifies seven emotions, phycological arousal and pleasure.

Finally, another solution is [15]. It proposes a solution to assess the capability of children having had diagnosed Autism Spectrum Disorders (ASD) to produce facial expression. This solution implements and uses Convolutional Neural Networks trained to detect micro facial expression. In this paper, a personalized and contextual statistic modelling of nonemotional face is formulated and used as a reference.

Nowadays, emotion recognition is a trend topic and it will still grow up in different fields. Emotion is an important factor in risky decision making and the relation between these two aspects is a crucial issue to be solved in future decision research. All these solutions try to solve a specific problem using this technology.

As above reported in the short state of art analysis, there are many examples of emotion recognition systems applied in different applicative scenario, but it is not easy to find them in the tourism sector. ETravel is an innovative solution that aims to support users in the in the hard task of choosing the perfect destination for a holiday travel. ETravel system exploits the AI, by using a Cloud-based platform, to predict an interesting location for the holiday based on user mood and reactions to different triggers.

\section{SYSTEM ARCHITECTURE}

The system architecture is based on different blocks. Each one is linked to the other, but all can be easily changed to integrate other service which cloud perform better results. The block-schema of the proposed solution is represented in Fig. 1.

It is composed of three main logical building blocks:

- ETravel Front-end app: it is a multi-platform application for mobile devices, like smartphones and tablets, which provides three main functionalities. Firstly, it proposes the user a kind of multi-sensorial questionnaire, i.e. a set of questions driven by images, text and sound stimuli, in order to stimulate user's emotional context during the travel planning. Secondly, by exploiting the device front camera, the app takes pictures of the user's face during the questionnaire and sends them to the FER (through the Cloud Server) in order to analyse the facial expression. Finally, the app acts as a terminal to show the customized travel solution proposed to the user after the evaluation of his/her emotional reaction to the questionnaire.

- Facial Emotion Recognition (FER) Service: it is an

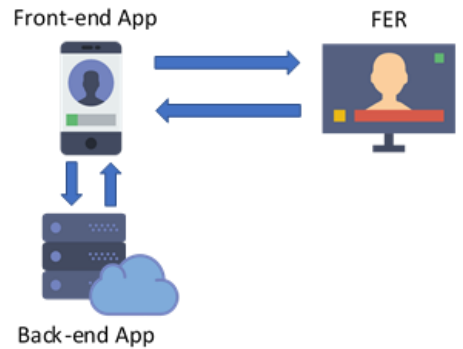

Fig. 1. Block diagram of the operational structure

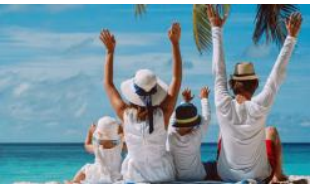

Family travel

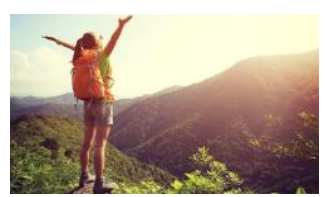

Lonely travel
Fig. 2. Trigger images examples

external Cloud Service that processes all the photos received from the app and it returns, for each photo, the result of the emotional analysis, by evaluating the likelihood of seven base emotion (happiness, angry, sadness, disgust, contempt, fear and surprise), and user's age and gender. 
- Cloud Back-end Server: It implements the business logic of the ETravel platform, basically a database with a set of labels linked to each "trigger" photo. Specifically, a trigger image, or sound, is a media used to stimulate a real and spontaneous reaction to a specific topic. Fig. 2 represents an example of two different images: the first one is about a family travel trigger, whereas the second one is about a lonely travel. By analysing user's reaction to a sequence of such images, the ETravel platform will try to compose a travel solution package with a high likelihood to meet user's expectations. The platform contains the following objects: filter functions with hard-coded labels, multimedia objects (images and sounds) and data objects representing questionnaires and destinations. Destinations are linked to specific labels. The Cloud Back-end also provides a set of APIs to request all media and text contents to show to the user.

\section{IMPLEMENTATION DETAILS}

\section{A. Front-end App}

The main block of the proposed system is the front-end App that includes several important features, such as user experience, user interface and provides all the operative functionalities.

As discussed in Section III, one of the primary goals of the front-end app is to stimulate user's mood and to capture his/her emotional reactions to specific "triggers". When the user starts the app, it downloads from the server a questionnaire with all the related photos, labels and sound. Labels are not static, so an administrator with access to the Cloud Back-end Server can upload and add new labels and changes settings of the app.

The main page of the app, shown in Fig. 3, presents four main elements:

- Photo trigger: it is a stock photo and it is used to stimulate user's facial expression

- Text trigger: it enforces the efficacy of the photo. It helps user to better understand the meaning communicated by the photo.

- Sound trigger: it is used to provide a most immersive tour with acoustic stimuli. For example, sound of traffic and cars can be associated to a big city photo.

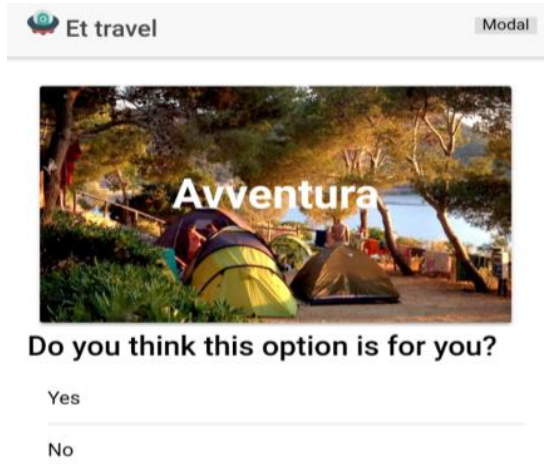

Fig. 3. Example of a page with triggers

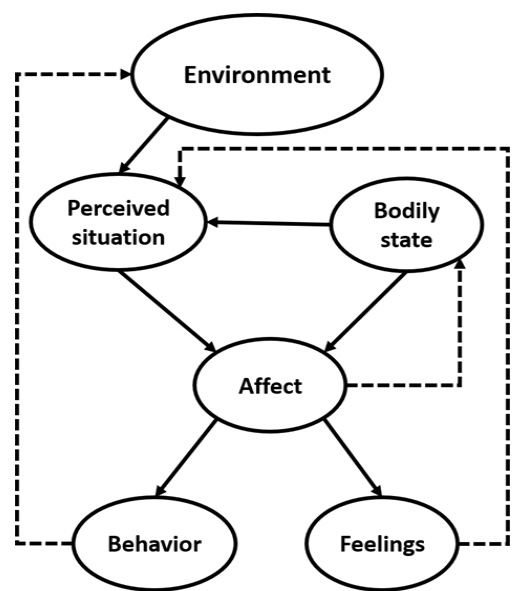

Fig. 4. Process of creation of an emotion/reaction flowchart

- Question form: it is a simple question form used to trigger the picture capturing of the user's face while he/she states his/her emotions.

All these kinds of triggers are used to establish an emotional link between users and app. Based on Pluchik [16], all emotions follow a process to manifest themselves Fig. 4. Specific environmental triggers activate cognitive perception. Later emotions appear and activate the reaction and then the feelings.

All trigger images are shown through an automatic slider.

Every time the user answers to the question form, a photo from the frontal camera is taken. This photo is sent to the Cloud Back-end Server. When the function returns the link to the resource, it is sent to the Facial Emotion Recognition Service. It provides emotion analysis, gender analysis and age analysis, as it will be explained in Section III.B. Results are processed to extrapolate information about user's mood.

The process in charge to generate labels to be sent to the server follows the logical flow shown in Fig. 5.

Each image is linked to a specific label. For example, as in

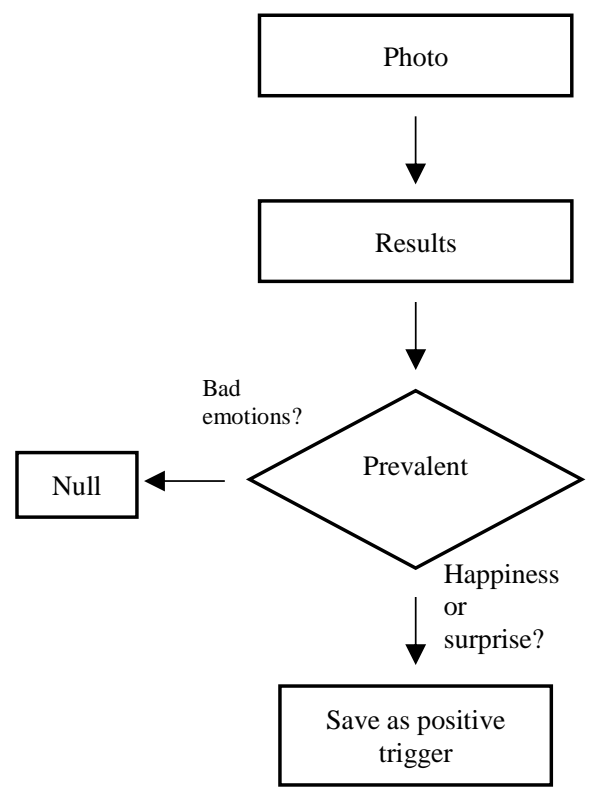

Fig. 5. Flowchart of creation of a label 


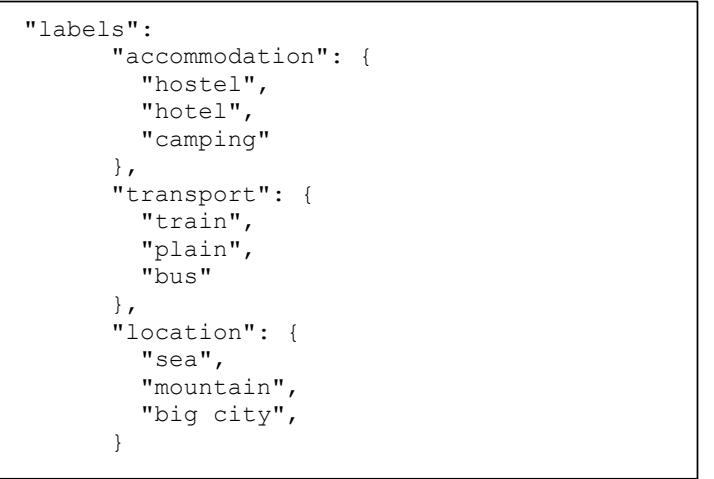

Fig. 6. Tree structure of labels

Fig. 3 image about camping is linked to label "adventure". When emotions results returned by FER are positive (happiness or surprise) the label liked to the trigger image is stored.

The labels are structured as in a tree as in Fig. 6. Each label can allow another, based on the result returned by FER in a specific user's session. This structure provides labels for: accommodation, transport and location, but the it can provide all kind of labels. For example, other labels could be about food, music, seasons, budget and so on. All labels can be linked to other labels, so to show results based on previous emotion analysis. In the following schema is shown our test structure.

When the questionnaire ends, and all labels have been generated, ETravel sends a request with them to the server. When the server provides a response, the app can optionally show the likelihood of the set of seven basic emotions for each image. By clicking "Destinations result" button, the user will access to all destinations matched with his inferred mood.

\section{B. Facial Emotion Recognition (FER) Service}

This is the module that provides emotion analysis, based on the Azure Face API [17]. By using Azure Face API and AWS S3 [18] Cloud Storage, real-time operations and data analysing from front-end app can be performed. Face API can perform different functions in addition to emotion, gender and age detection such as:

- Face identification

- Face comparison

- Face pose detection,

- Detection of characteristics face (makeup)

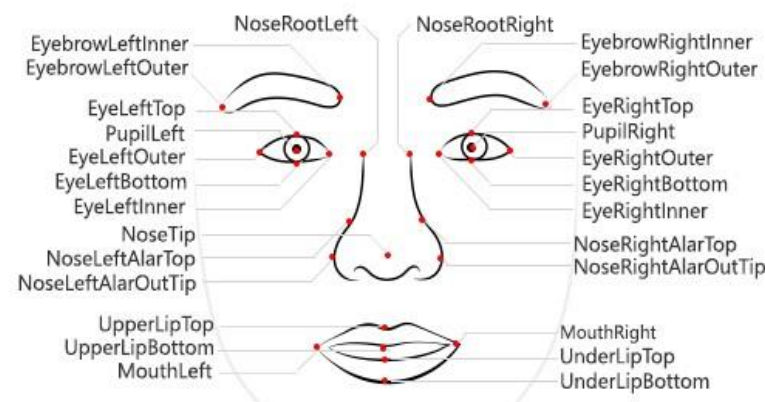

Fig. 7. Azure Face Api facial landmarks
Fig. 7 shows the main face landmarks used by the Face API to perform its analysis.

Face API ensures a better result with higher face image quality, which means better detection and recognition precision. The recommended resolution and position of good photos are: frontal, clear, and face size of 200x200 pixels. It supports JPEG, PNG, GIF (the first frame), and BMP format. The allowed image file size is from $1 \mathrm{~KB}$ to $6 \mathrm{MB}$. Attributes returned by Azure Face API are: age, gender, headPose, smile, facialHair, glasses, emotion, hair, makeup, occlusion, accessories, blur, exposure and noise.

FER Service provides emotions analysis for seven different emotions: joy, fear, sadness, happiness, contempt, disgust and surprise. It provides also gender and age information. This kind of information can be useful to develop a more efficient destination matching function.

The Azure Face API returns results to a photo like Fig. 8part A in JSON format. The structure of the results is like in the example showed in Fig. 8-part B.
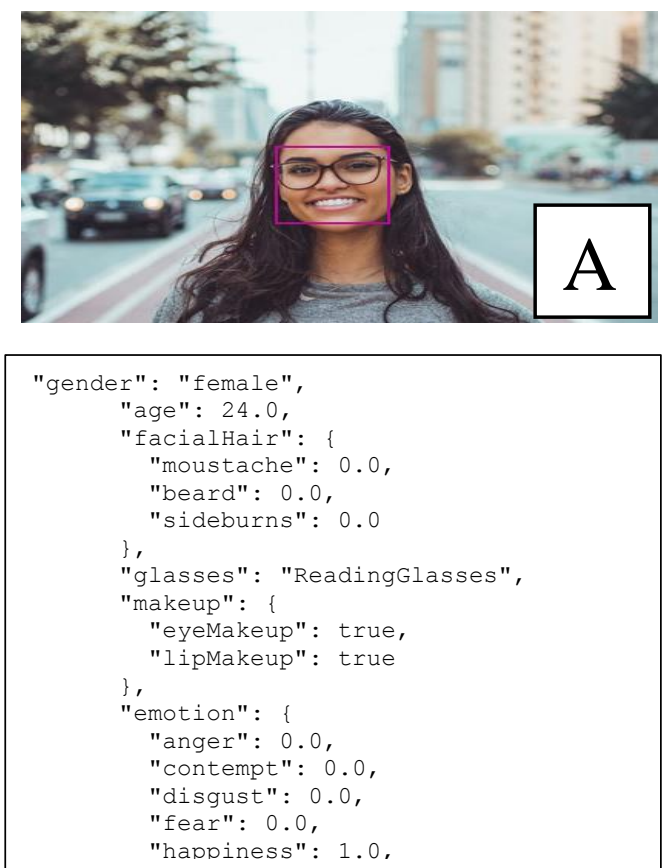

Fig. 8. Example of a result returned by Azure to the photo above

\section{Cloud back-end Server}

This module provides database interactions. Database is structured to store:

- Destinations: it is a solution for a travel. It contains all data about a destination.

- Labels: it is a tag for destinations. All destinations are related with multiple labels, based on type of destination. For example, a label is a tag about how family friendly a city is. An example of labels and destinations relation is shown in Fig. 9. As it can be seen, there are two different destination labelled with their most relevant attributes. 


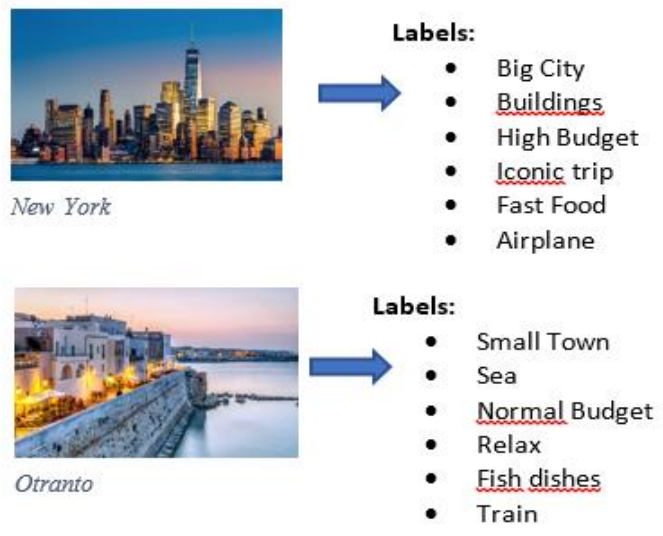

Fig. 9. Example of labels linked to different destinations

All this data can be generated in two different ways. The first one is to upload labels and destination manually, by querying the database with data passed by us, the second one is an automatic way.

This way is about to collect automatic labels for a destination, by using text-analysis service to detect main words about it into internet and generating automatic labels that could increase the database and make it more efficient.

When the user's questionnaire ends, the app proceeds to retrieve all destinations matched. The system has generated for each "trigger" image a label based on flow in Fig. 4. At the end all labels are sent to the server by a GET HTTP function. The server reads all labels passed through REST APIs and it matches destinations and labels received to get the most accurate results for the user. Then it returns all destinations in, found in JSON format to client app to show them to the user. By updating database, it is possible to update user tour and to make it dynamic. New trigger can be added, so to add new filters and sub filters.

All media processed and analysed are stored on S3 by Amazon AWS. It allows us to store:

- User photos: these photos are stored temporary in Cloud. When upload ends, it returns a link. This link is sent to Azure Face API to analysis user's mood.

- Trigger images: these images are used to encourage user's mood. They communicate a direct message.

- Sounds: they are sounds related to images.

\section{SYSTEM VALIDATION}

For this work is very important to validate:

- Results returned by the FER service to a set of images: two different databases have been used to get information about Azure Face Api to analyse images with posed and not posed expressions. The results are been compared to check its capability to detect emotions in different cases. Both databases have images with hard labels, so it was easy to check the results.

- The ETravel system: this part of the project is the app which integrates the FER module. It provides the innovative solution to emotional tourism by interreacting with different modules. This validation is about to get information about app results. We choose a set of people's faces from $\mathrm{CK}+$ and we predict destinations which should be the most accurate of all from database destinations. Then we simulate the user's use case. In the final step the results have been compared with the predicted destination.

The results of this validation are shown in Section V.A.

\section{A. FER}

Before implementing the FER Service, the Azure Face APIs have been evaluated to check whether the platform is useful for the proposed solution. Two different dataset of face images have been used: the first one, a handmade database called Db FI (Internet Face database) and the second one, the well know $\mathrm{Ck}+[19]$ [20] database.

The results from each database used and the comparison between the two results are shown in Fig. 10.

Db FI is composed of face images taken all around the Internet. Table I shows the number of images for each type of emotion used to test the platform. Selected images were already labelled, from different Internet sources, and depicted children, women and men faces with specific emotions. The image labels have been useful to check results returned from the platform. This database groups people's images with a strong emotional component on their faces. Most of them are posed expression and not natural. The result got from this database has been for qualitive comparison with $\mathrm{CK}+$ result. This database has been created just to check results between images with explicit expression and images non-posed and natural expression from $\mathrm{CK}+$. All images were free or loyalty free and some of them had a problem with a watermark. That is because some watermarks were placed on the face and they could affect negatively some landmarks and consequently the

face analysis. Nonetheless, this dataset provided important results. As shown in Table II, from a detection accuracy point of view, the most relevant emotions are happiness, surprise, sadness and anger. The first two are very important for the ETravel purpose, because when the system detects these emotions, it generates the filters to be sent to the server. The second one, instead, are the most frequent negative emotions.

Positive emotions have a rate of wrong result around of 5$10 \%$. This range is considered acceptable for the needed requirements. On the contrary, sadness and fear have a rate around $15 \%$ of negative results. This dataset returned good results for positive emotions, whereas performances were quite poor regarding negative emotions. Therefore, the CK+ dataset was evaluated.

CK+. The Cohn-Kanade AU-Coded Facial Expression Database has been expressly created for research in automatic facial image analysis and synthesis and for perceptual studies. Cohn-Kanade is available in two versions and a third is in preparation. $\mathrm{CK}+$ includes both posed and non-posed (spontaneous) expressions and additional types of metadata.

With this dataset, all emotions' detection accuracy increased their percentage of success Table II. The only one to decrease 


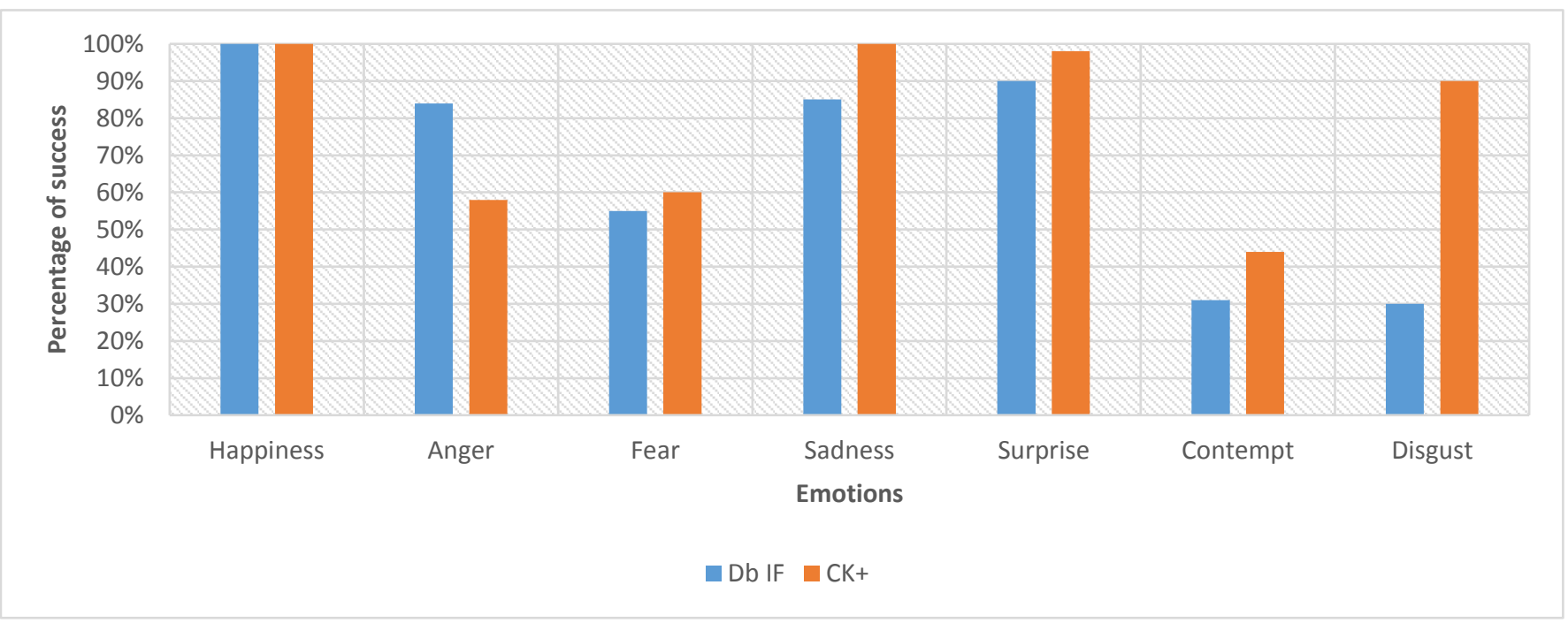

Fig. 10. Comparison between emotion analysis positive results returned by Azure for each image in DBIF and CK+

the success rate was anger.

Based on these tests, positive emotions had a very low rate of false-positive. Only the surprise emotion had some falsepositive with negative emotions. Instead, sadness, anger, fear, contempt and disgust had falser-more positive with happiness, surprise and bad emotions.

In conclusion, these tests demonstrate that the Face API is a good and reliable platform to integrate into the proposed ETravel platform.

\section{B. Functional Validation of the ETravel App}

ETravel provides a simple a clear user interface. It wants to help user to find the best destinations based on user's mood and reactions, so user's actions inside the app are very limited.

The main page is like in Fig. 11. It shows how homepage appear to the user. The homepage has only a slider for images trigger and a button to send a request to the server.

After homepage, each page is composed by:

- Text trigger

- Sound button. It is used to pause or play background sound

- Image trigger

- Simple question

The question is always the same and very short because the user has not to get distracted by it. The answer should be the

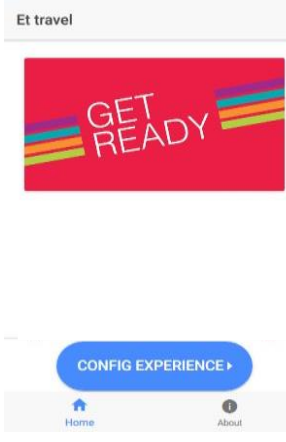

Fig. 11. Home page of ETravel most natural and spontaneous possible. A long or different question could deface spontaneous reaction.

Fig. 12 shows the main steps which every user must follow to use the app. The main steps of the app are:

- The first one is the step one of Fig. 12 step A. This step is about app configuration. The app when the user clicks on "Config" send by REST API HTTP GET request to the server a request for all media to load into the app all the media and parameters necessary. All dynamic resources such as trigger, audio, text, are stored on the server. This is because it is important to make the app as much as possible easy to update, so if new triggers need to be added, we need to update only the server and not the app at all.

- The second step is about user interaction with the app as in Fig. 12 step B. A new page is shown. This one has the fields discussed above. The user with a pair of headphones can join the questionnaire. A set of images will be shown inside the slider every time the

TABLE I

Percentage of SucCESS ReSUlt FROM AZURE TO IMAGE FROM CK+ AND DBIF

\begin{tabular}{|c|c|c|c|c|c|c|c|}
\hline & Happiness & Anger & Fear & Sadness & Surprise & Contempt & Disgust \\
\hline $\begin{array}{c}\mathrm{Db} \\
\mathrm{IF}\end{array}$ & $100 \%$ & $84 \%$ & $55 \%$ & $85 \%$ & $90 \%$ & $31 \%$ & $30 \%$ \\
\hline $\mathrm{CK}+$ & $100 \%$ & $58 \%$ & $60 \%$ & $100 \%$ & $98 \%$ & $44 \%$ & $90 \%$ \\
\hline
\end{tabular}

TABLE II

NUMBER OF IMAGES FOR EACH EMOTION IN CK+ DATABASE AND DBIF DATABASE

\begin{tabular}{|l|c|c|c|c|c|c|c|}
\hline & Happiness & Anger & Fear & Sadness & Surprise & Contempt & Disgust \\
\hline $\begin{array}{l}\mathrm{Db} \\
\mathrm{IF}\end{array}$ & 39 & 24 & 36 & 48 & 50 & 32 & 23 \\
\hline $\mathrm{CK}+$ & 65 & 45 & 25 & 28 & 82 & 18 & 55 \\
\hline
\end{tabular}

user answers the question. Meanwhile a song, related to the image shown into the slider, will play in background. Each time the user answers the question, the system takes a photo of him. This photo is taken in background and then a 
preview is shown. The photo is temporary stored on S3, then the link returned is passed to Azure to analyse the user's reaction. The result returned from Azure is processed to generate a label, based on process in Fig. 4. When the process ends, the result is stored and not shown in the meanwhile. It is shown at the end of the questionnaire.

- The third step is when the user ends the questionnaire as in Fig. 12 step C. The system sends by the REST API a request with all labels generated to the server. The server sends back all destinations found. All results got from Azure are shown for each photo. Each photo has value for all seven emotions and neutrality of user's reaction. The user can scroll them as in red box of the figure.

- The last step is about to get all destinations as in Fig. 12 step D. When the user proceeds to destination's page, he can view all destinations found.

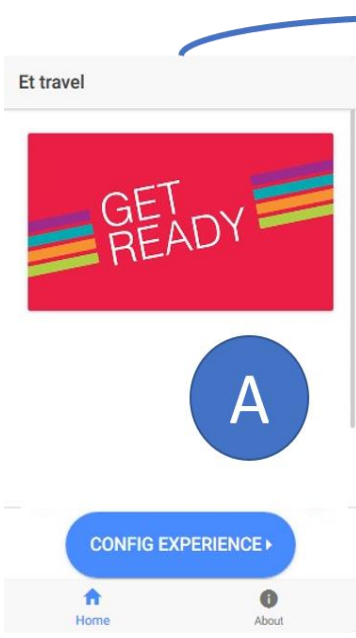

prediction. We succesfully intragtes diffent modules in the app. The most import module is the FER, which uses Azure return us positive results from this work.

In the future it could integrate a service to map automatic destination and labels to increase database destinations and database labels. New technology could be used to perform facial expression analysis, for example with trained microexpression neural network to improve the algorithm and return results closer to the user's mood. In the next step, it would be useful to integrate a better labels generation process. In this works a static structure of label has been used. A dynamic version could improve images trigger shown process to the user by excluding images related to other images, based on the results of previously shown images. By integrating all these new features, it will be possible to define a real-time assistant able to suggest other activities in loco, for example which activity is the best for the user or which restaurant is the best based on its menu.

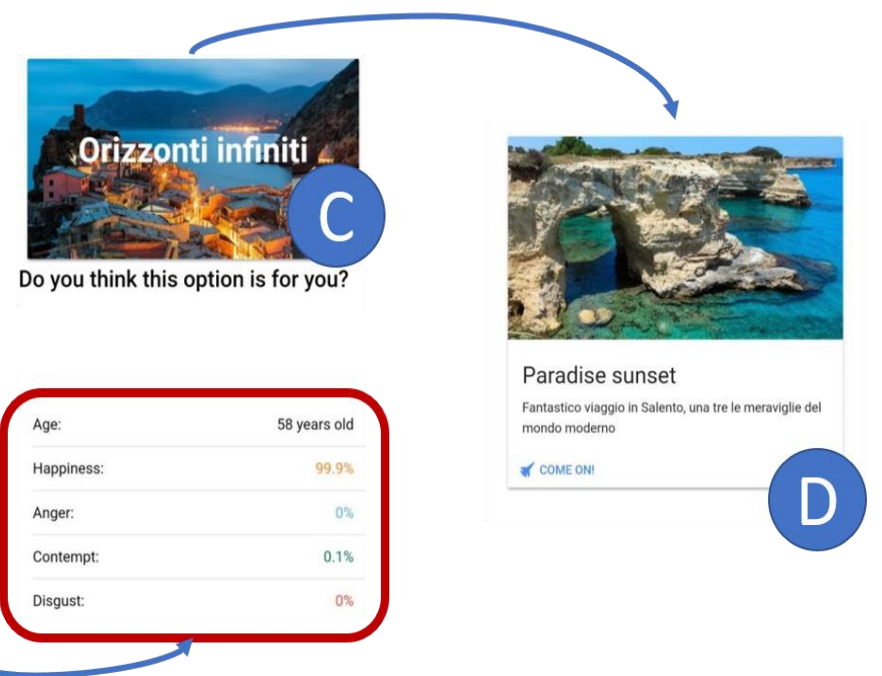

Fig. 12. All steps of a user's uses case

In this test we used different photo of the same person in different emotional states. Before we decided to choose as destination "Paradise Sunset", a destination with the following labels linked to:

- Relax: a label about a destination to join relax

- Skipping town: a label about a destination far from the hustle and bustle of the city

- Unending horizon: a label about a coast

We passed images with positive emotions to the trigger images related to these labels, and bad one to the others. The result was correctly. It returns what we expected "Paradise Sunset”.

\section{DISCUSSION AND CONCLUSIONS}

This work proposes an innvoative solution to planning a travel. In tourism there is not any solution which tries to implement emotion analysis with AI. This solution tries to integrate an innovative service to the tourism filed.

By testing this app we got good results about destionations
In this version, photo from $\mathrm{CK}+$ has been used to test the system, but in the future the next step should be to test the system with real people's face and check the result in real life.

\section{REFERENCES}

[1] Ekman, P. (2005). Basic Emotions. In Handbook of Cognition and Emotion (eds T. Dalgleish and M. J. Power). 45-60 doi: 10.1002/0470013494.ch3

[2] Deshwal, P. (2015) Emotional Marketing: Sharing the Heart of Consumers. International Journal of Advanced Research in Management and Social Sciences, 4, 254-265.

[3] "Just do It Nike." [Online].

Available: https://news.nike.com/featured_video/just-do-it-dream-crazyfilm. [Accessed: 15 Dec. 2018].

[4] "Real Beauty Dove." [Online].

Available: https:/www.dove.com/us/en/stories/campaigns/real-beautysketches.html. [Accessed: 15 Dec. 2018].

[5] Bigné, Enrique \& Andreu, Luisa. (2004). Emotions in Segmentation: An Empirical Study. Annals of Tourism Research. 31. 682-696. 10.1016/j.annals.2003.12.018.

[6] Shichuan Du, Yong Tao, and Aleix M. Martinez Compound facial expressions of emotion, 2014 
[7] Centobelli, P., \& Ndou, V. (2019). Managing customer knowledge through the use of big data analytics in tourism research. Current Issues in Tourism, 1-22.

[8] R. Leijdekkers et al., "CaptureMyEmotion: A mobile app to improve emotion learning for autistic children using sensors", Proc. Int. Symp. Comput. -Based Med. Syst., pp. 381-384, 2013

[9] "Affectivia." [Online]. Available: https://www.affectiva.com/. [Accessed: 15 Dec. 2018].

[10] Y. Seanglidet et al., Mood prediction from facial video with music "therapy" on a smartphone, London: IEEE, 2016.

[11] P. Ekman, W. V. Freisen, and J. C. Hager, Facial Action Coding System, vol. 160, 2nd ed. Salt Lake City, UT, USA: Research Nexus eBook, 2002

[12] Bouzakraoui, M.S., Sadiq, A., Enneya, N.: Towards a framework for customer emotion detection. In: IEEE/ACS 13th International Conference of Computer Systems and Applications (AICCSA), Agadir, pp. 1-6 (2016)

[13] José Mennesson et al., Faces and thoughts: An empathic dairy, Ljubljana, Slovenia: IEEE, 2015

[14] Ivan, A. L., Begoña, J. N., \& So-Yeon, Y. O. O. N. Identifying customer's emotional responses towards guest-room design by using facial expression recognition, in hotel's virtual and real environments. 2018

[15] Leo, M.; Carcagnì, P.; Distante, C.; Spagnolo, P.; Mazzeo, P.L.; Rosato, A.C.; Petrocchi, S.; Pellegrino, C.; Levante, A.; De Lumè, F.; Lecciso, F. Computational Assessment of Facial Expression Production in ASD Children. Sensors 2018, 18, 3993

[16] Plutchik, R. (1991). The emotions: University Press of America.

[17] "Azure Face Api." [Online]. Available: https://azure.microsoft.com/enus/services/cognitive-services/face/. [Accessed: 15 Dec. 2018]

[18] "AWS S3." [Online]. Available: https://aws.amazon.com/it/s3/. [Accessed: 15 Dec. 2018].

[19] Kanade, T., Cohn, J. F., \& Tian, Y. (2000). Comprehensive database for facial expression analysis. Proceedings of the Fourth IEEE International Conference on Automatic Face and Gesture Recognition (FG'00), Grenoble, France, 46-53.

[20] Lucey, P., Cohn, J. F., Kanade, T., Saragih, J., Ambadar, Z., \& Matthews, I. (2010). The Extended Cohn-Kanade Dataset (CK+): A complete expression dataset for action unit and emotion-specified expression. Proceedings of the Third International Workshop on CVPR for Human Communicative Behavior Analysis (CVPR4HB 2010), San Francisco, USA, 94-101. (Gretzel,2011; Werthner, 2003; Sigala and Chalkiti,2014).

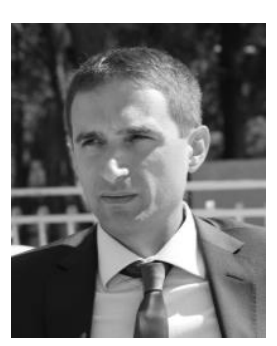

Luigi Patrono received his MS in Computer Engineering from University of Lecce, Lecce, Italy, in 1999 and $\mathrm{PhD}$ in Innovative Materials and Technologies for Satellite Networks from ISUFIUniversity of Lecce, Lecce, Italy, in 2003. He is an Assistant Professor of Network Design at the University of Salento, Lecce, Italy. His research interests include RFID, EPCglobal, Internet of Things, Wireless Sensor Networks, and design and performance evaluation of protocols. $\mathrm{He}$ is Organizer Chair of the international Symposium on RFID Technologies and Internet of Things within the IEEE SoftCOM and IEEE SpliTech conferences. He is author of about 120 scientific papers published on international journals and conferences and four chapters of books with international diffusion.

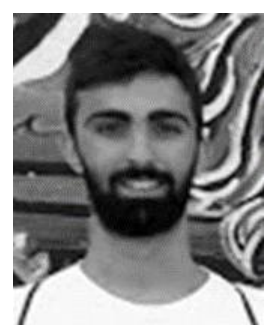

Luca Podo is a graduated in Computer Engineering at the University of Salento. He got a bachelor's degree about "Design and implementation of a multi-platform smartphone application for emotional tourism" at the IDA Lab at the University of Salento. His subjects of studies are IoT, Artificial Intelligence and Computer Vision.

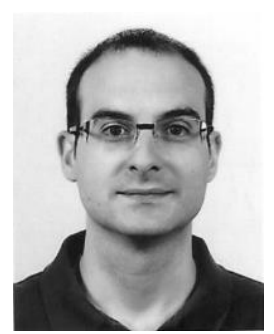

Piercosimo Rametta received the Master's degree in Computer Engineering with honours at the University of Salento, Lecce, Italy, in 2013. His thesis concerned the definition and implementation of a novel mash-up tool for Wireless Sensor Networks' configuration. From November 2013 to October 2018 he collaborated with IDA Lab IDentification Automation Laboratory at the Department of Innovation Engineering, University of Salento. His activity focused on the definition and implementation of new mash-up tools for managing smart environments based on Wireless Sensor Networks and Internet of Things. 